Original Contribution

\title{
DIAGNOSTIC ACCURACY OF MRCP IN EVALUATION OF PERIAMPULLARY DUODENAL DIVERTICULA - PROSPECTIVE STUDY
}

\author{
P. Getsov ${ }^{1}$, S. Mitova-Siminkovitch ${ }^{2}$, S. Dineva ${ }^{3 *}$, B. Vladimirov ${ }^{2}$ \\ ${ }^{1}$ Department of Diagnostic Imaging at University Hospital "Queen Joanna-ISUL", Sofia, Bulgaria \\ ${ }^{2}$ Department of Gastroenterology, University Hospital "Queen Joanna-ISUL", Sofia, Bulgaria \\ ${ }^{3}$ Department of Diagnostic Imaging, Medical Institute of Ministry of Interior, Sofia, Bulgaria
}

\begin{abstract}
Background and Objectives: Duodenal diverticula are a common finding in patients with biliary tract disorders. The objective of this study is to assess the diagnostic value of the magnetic resonance cholangiopancreatography (MRCP) in case of periampullary duodenal diverticula (PAD) using endoscopic cholangiopancreatography (ERCP) as a reference method.

Methods: The study population included 350 patients with known or suspected diseases of the biliary tract, liver, and pancreas. Two radiologists interpreted the image quality and the presence of PAD in agreement .

Results: In cases with a single PAD, MRCP had sensitivity $81.2 \%$, specificity $98.6 \%$, positive predictive value $90.9 \%$, and negative predictive value $97 \%$, compared to ERCP. In the case with two PAD, MRCP had sensitivity $40 \%$, specificity $99.7 \%$, positive predictive value $66.7 \%$ and negative predictive value $98.9 \%$. The average volume of PAD was $3.25 \mathrm{~cm} 3$. In this study group patients with PAD were on average 10 years older than those without PAD $(<0.001)$. There was also found a significant association of PAD with choledocholithiasis $(p=0.009)$ and cholecystitis $(p=0.002)$.

Interpretation and Conclusions: In conclusion, MRCP could be used as a non-invasive diagnostic modality with acceptable sensitivity and specificity in patients with suspected PAD and biliary tract diseases.
\end{abstract}

Key words: MRCP, ERCP, duodenal diverticulum, cholecystitis, choledocholithiasis, bile ducts.

\section{INTRODUCTION}

The duodenum ranks second in the frequency of diverticula in the gastrointestinal tract after the colon. Most of the small bowel diverticula are located in the duodenum. Approximately $80 \%$ of the primary duodenal diverticula are found in the retroperitoneal space, median to the second part of the duodenum, at a distance of up to $20 \mathrm{~mm}$ from the ampulla of Vater. These are also known as periampullary diverticula (PAD). The congenital weakness of the duodenal wall is thought to explain the high incidence in the area where the bile and pancreatic ducts enter the lumen. The pathophysiological mechanism of occurrence included both traction and pulsion.

Correspondence to: Dr. Stoyanka Dineva; dineva_g@abv.bg 359-887-520748 MD, PhD, Radiologist, Department of Diagnostic Imaging, Medical Institute of Ministry of Interior, Sofia. 79 Scobelev St, 1527 Sofia, Bulgaria
Periampullary diverticula were first described by Chomel in the far 1710 (1). Since then, PAD was considered as an anatomical variety (2-10). In 1908, Rosenthal reported three cases of a periampullary diverticulum in combination with biliary obstruction (11). JT Case made the first radiological demonstration in 1913 (12). Since then many authors have been focusing an interest in the prevalence, diagnostic modalities and the relationship between PAD, and the diseases of the biliary system.

The objective of this study was to evaluate the diagnostic value of MRCP in detecting PAD and their relationship with gender, age and biliary tract diseases.

\section{MATERIAL AND METHODS \\ 1. Design and Patients:}

This longitudinal, prospective study was conducted between April 2010 and April 2014 in the Clinical Centre of Gastroenterology at 
the University Hospital , Queen JoannaISUL", Sofia. Overall 350 consecutive patients with known or suspected hepato-billiary or pancreatic disease were included after obtaining of informed consent in accordance with the Declaration of Helsinki, its amendments and the GCP principles. All patients underwent ERCP and were next scheduled for MRCP. Table 1 presents the characteristics of the final analyzed patient cohort.

Table 1. Characteristics of the analysed patient cohort

Patient characteristics

$\mathrm{N}$

\begin{tabular}{l|l}
\hline Gender & \\
\hline Male & 172 \\
\hline Female & 178 \\
\hline Age & \\
\hline mean & bb \\
\hline SEM, SD... & aa \\
\hline Patients with one PAD & 48 \\
\hline Patients with more than one PAD & 5 \\
\hline Reason for ERCP referral* & \\
\hline Gallbladder disease & 232 \\
\hline Intra- and extrahepatic bile duct pathology & 224 \\
\hline Pancreatic disease & 63 \\
\hline
\end{tabular}

* number exceed total number of patients due to combined pathology

\section{Magnetic Resonance Imaging:}

All patients underwent MR imaging (GE Signa XDe $1.5 \mathrm{~T}$ machine with 8ch body upper coil) after the ERCP as the last was used as a reference standard. The radiologist was initially blinded for the ERCP results. MR acquisition involved a 3D MRCP ASSET (TR $5455 \mathrm{~ms}$, TE $785,7 \mathrm{~ms}$ ), T2 FIESTA in coronary plane (TR $6,8 \mathrm{~ms}$ TE $3.0 \mathrm{~ms}$ ), T1 DualEcho (TR 125ms TE 2,37 / 4,71 ms) and T2ssFSE Fat Sat (TR 3818ms TE 99,52ms). Image analysis was performed on the workstations of the manufacturer of the MR scanner (GE Advanced Workstation, software v. 4.4 and 4.5). 3D Synchro View software was used to compare the signal characteristic of a pathological process in the different sequences. PAD dimensions and volume were measured using two mutually perpendicular diameters on the axial plane and the maximum caudalcranial diameter of PAD.

The transverse and anterior-posterior diameters were measured on images from T2ssFSE Fat Sat series and, if necessary, T1 DualEcho FSPGR ASSET images in the axial plane. The caudal-cranial diameter was determined on 3D
MRCP ASSET and T2 FIESTA in the coronal plane. In case of more than one PAD, only larger was measured. In both cases, they were closer to ampulla of Vater.

3. Statistical Analysis - was performed using SPSS 16.0 (Chicago, IL) software. The data were described by the mean, $95 \%$ confidence interval (CI) of the mean, standard deviation (SD) and standard error of the mean (SEM). Alpha level of 0.05 was accepted as significant.

\section{RESULTS}

The study cohort consisted of 350 patients (172 males, 178 females), mean aged $56.83 \pm$ 15.92 years (range 15 - 86 yrs). The gender difference in the study group was not statistically significant, $p>0.05$. Periampullary duodenal diverticula were detected during the ERCP in 53 patients (15.1\%). Forty eight patients had single PAD (13.7\%), while the rest 5 patients $(1.4 \%)$ had two.

The incidence of PAD was numerically higher in women (60\%) than in males $(40 \%)$, without reaching significance $(p>0.05$, Pearson Chi- 
Square test). Interestingly, patients with PAD were about 10 years older than those without PAD $(\mathrm{p}<0.05)$.

\section{Pad And Bile Ducts Diseases.}

The initial diagnosis for ERCP referral was gallbladder disease, intra- and extra hepatic bile duct pathology or pancreatic disorders (Table 1).

Bile duct diseases in the studied cohort included choledoholithiasis, dilatation of an extrahepatic duct not caused by tumor or lithiasis, benign stricture, tumors and biliodigestive fistulas. In the PAD group, 71,7 $\%$ of patients had evidences of a disease of the intra- and extrahepatic bile ducts while these were only found in $62.6 \%$ of patients without PAD. Choledocholithiasis was found in $30.2 \%$ of patients with PAD and only in $14,5 \%$ of those without PAD. We also found that presence of PAD correlated with evidence of choledocholithiasis $(\mathrm{p}=0.009)$.

\section{PAD And Gallbladder Diseases}

Gallbladder disease in the studied cohort included cholecystitis, carcinoma of the gall bladder, fistulas and infiltration of the bladder by adjacent neoplastic process. In patients with PAD, gallbladder disease was found in $83 \%$ while only in $63.3 \%$ of patients without PAD. Similarly, in patients with PAD calculous cholecystitis was found in $75.5 \%$, while only in $54.5 \%$ of patients without diverticula. We found that the presence of PAD correlated with calculous cholecystitis $\quad(p=0.002)$. Unfortunately, the limited number of cases did not allow us to perform multivariate regression to assess the potential confounding due to gender, age, concomitant disease, etc.

\section{PAD And Pancreatic Pathology}

Among the studied cohort, 63 patients were referred for ERCP and respectively for MRCP because of a pancreatic disease in addition to a bile duct pathology. Most commonly were seen patients with chronic pancreatitis $(n=45)$ and benignant or malignant tumors. We identified seven cases of PAD among patients with pancreatic disease (4 with pancreatitis and rest with cancer). Within this subgroup, only 25 patients had no accompanying pathology of biliary tract and only 23 had no accompanying pathology of the gallbladder. Fifteen patients had neither bile duct nor gallbladder disease, and among them one patient had PAD and chronic pancreatitis.
GETSOV P., et al.

Comparison of the diagnostic yield of MRCP compared to ERCP as a reference standard for diagnosing PAD.

In the study, group ERCP demonstrated PAD in $15.1 \%$ of patients. Single diverticulum was found in $48(13.7 \%)$ and two diverticula in 5 patients (1.4\%). For comparison MRCP detected PAD in $13.2 \%$ of patients - single diverticulum was found in $45(12.8 \%)$ and two $\mathrm{PAD}$ in 2 patients $(0.6 \%)$.

Using an MRCP modality we did not find evidence for PAD in 294 patients, which was confirmed by ERCP in $98.7 \%$ of cases. In 4 patients $(8.9 \%)$ we detected single PAD on MRCP while it was not confirmed in the ERCP series. In all 39 patients in whom MRCP detected a single diverticulum, this was confirmed by ERCP. In $81.3 \%$ of cases with one PAD, both methods agreed as a result. In 9 patients, we found no PAD on MRCP while it was evident on the ERCP. Overall in $18.8 \%$ of the cases, MRCP had a false negative result for a single diverticulum.

In 4 patients who had two PAD, the two methods demonstrated worse correlation. In 2 patients MRCP showed two PAD, which was evident also on ERCP series. In other two patients we found one PAD on MRCP, while there were two PAD on ERCP. One patient with two evident PAD on ERCP was not confirmed on MRCP.

We have repeated the analysis of MRCP images after unblinding the radiologist for the ERCP results in the cases where the two methods did not match. Thus we further identified 4 patients with a single PAD. One PAD was also detected in a patient with $2 \mathrm{PAD}$ on ERCP. In the 4 patients with a false positive diagnosis of PAD on MRCP we further diagnosed small pseudocysts on the head of the pancreas (additional CT imaging was required).

Since the results of the descriptive analysis showed large differences in the presence of one or two PAD, they were subsequently independently examined.

For this purpose, the MRCP results were directly compared to ERCP, and sensitivity, specificity, positive and negative predictive values were examined. True positive were cases with single PAD on ERCP with MRCP detection. False-positive were the cases where patients had no PAD on ERCP, but MRCP was 
analyzed as having one. True negative were cases had no PAD neither on ERCP nor on MRCP. False negative were the cases where the ERCP detected PAD, but MRCP does not imaged them.

The results of the analysis of sensitivity, specificity, PPV and NPV are summarized in table II. Additionally, the ability of MRCP to detect the presence of two periampullary diverticula was investigated (Table 2). The results of both methods were subjected to the Pearson Chi-Square test $\left(\mathrm{X}^{2}\right)$. It was found that in the presence of two PAD between them there was a statistically significant correlation, $\mathrm{p}<0.05$.

Table 2. MRCP versus ERCP in establishing a single PAD and two PAD.

\begin{tabular}{lllll}
\hline & Sensitivity $\%$ & Specificity $\%$ & $\begin{array}{l}\text { Positive } \\
\text { Predictive value } \\
\%\end{array}$ & $\begin{array}{l}\text { Negative } \\
\text { Predictive value }\end{array}$ \\
\hline Single PAD & 81.25 & 98.66 & 90.71 & 97.03 \\
\hline Two PAD & 40.00 & 99.66 & 66.66 & 98.99 \\
\hline
\end{tabular}

In addition, ROC curve analysis found that MRCP has very good diagnostic value for identifying PAD as compared to ERCP as a reference standard - AUC 0.863 (SE 0.109), $\mathrm{p}=0.005(95 \%$ CI $0.649-1.000)$.

Accuracy of the MRCP for PAD is $86.8 \%$ (87.2\% for $\mathrm{s}$ ingle $\mathrm{PAD}$ and $99.4 \%$ for two PAD).

\section{Volume Of PAD}

Our MRCP protocol allowed measurement of the PAD dimensions and calculation of their volume. The mean transverse diameter of the PAD was $16.85 \mathrm{~mm}$ (95\% CI 15.47-18.24 $\mathrm{mm})$. The mean anterior-posterior diameter of the PAD was $16.75 \mathrm{~mm}$ (95\% CI 15.14-18.36 $\mathrm{mm})$. In this patient cohort, the caudal-cranial diameter of the PAD was longest with a mean value of $18.71 \mathrm{~mm}(95 \%$ CI $17.15-20.27 \mathrm{~mm})$

- Table 3.

Table 3. Average PAD diameters

\begin{tabular}{llllll}
\hline Diameters & \multicolumn{2}{l}{ Mean value } & \multicolumn{2}{l}{ Standard deviation } & 95\% CI Standard error \\
\hline $\mathrm{D} 1(\mathrm{~mm})$ & 16.85 & 4.758 & $15.47-18.24$ & 0.69 & \\
\hline $\mathrm{D} 2(\mathrm{~mm})$ & 16.75 & 5.541 & $15.14-18.36$ & 0.80 & \\
\hline $\mathrm{D} 3(\mathrm{~mm})$ & 18.71 & 5.371 & $17.15-20.27$ & 0.77 & \\
\hline
\end{tabular}

The average volume of PAD in our group was calculated $3.25 \pm 2.58 \mathrm{~cm}^{3}(95 \%$ CI $2.5-4$ $\mathrm{cm}^{3}$ ).

We did not found significant correlation between the age of the patient and the volume of the diverticula.

\section{DISCUSSION}

Over the last two decades, the MRCP has gained increasing importance in the diagnosis of diseases of the biliary tract and the pancreas, even with regard to radiation protection of the patient (13). Invasive techniques such as ERCP and PTC were displaced in routine diagnostics by MRCP. However, diseases of the pancreas are relatively rare indication of performing an MRCP. From another point of view, pancreatitis, including complicated cases, is among the most frequent indications for MRCP. There were only a few studies regarding the place of MRCP for the diagnosis of $\operatorname{PAD}(14,15,16)$. Morita et al $(15)$ were investigated the sensitivity, specificity and accuracy of three MRCP techniques: 3D TrueFISP MRCP, 2D RARE MRCP, and 2D TSE MRCP. For 3D TrueFISP MRCP, these indicators were $61.9 \%, 85.7 \%$ and $73.8 \%$, respectively. For 2D RARE sensitivity, specificity and accuracy are respectively $0.0 \%$, $100 \%$ and $50 \%$, and for 3D TSE MRCP were $9.5 \%, 100 \%$ and $54.8 \%$.In our study we used 3D MRCP FRFSE ASSET sequence (3D Fast Recovery Fast Spin Echo with array spatial sensitivity encoding technique). This sequence allowed reduction of motor artefacts and takes less time (3-4 minutes at $3 \mathrm{~mm}$ thickness of the slices obtained). The technique used by Morita et al 3D TrueFISP MRCP technique was very 
close in technical terms. It was characterized by high image acquisition speed and less impact of the patient movements. Both techniques were particularly suitable for patients with breathing difficulties. Our results correspond to these 3D TrueFISP MRCP series of Morita et al. We also found that in cases with a single PAD MRCP has given very good results-. The sensitivity of the method compared to ERCP was $81 \%$ and the specificity was $99 \%$. The positive predictive value was $91 \%$ and the negative was $97 \%$. Far less was the sensitivity of MRCP compared to ERCP in patients with two PAD - only $40 \%$. Although the specificity and the negative predictive value in the cases of two diverticula were relatively high (99.7 and $98.9 \%$ respectively), the positive predictive value remained relatively low $-66.6 \%$.

The publications on the accuracy of imaging diagnostic methods for determining the size and volume of PAD were referred mainly to MDCT (multidetector CT) and MRCP $(5,14$, 17). Perdikakis et al.(16) explored the role of the MDCT and MRCP in the diagnosis of PAD in patients with perforated diverticulum, pancreatitis, acute cholecystitis, dilated biliary tract and acute pain after feeding. The mean diameter along the long axis of the diverticula in the study group was $2.67 \mathrm{~cm}(0.96-4.9 \mathrm{~cm})$. MDCT allowed the detection of diverticula in both axial and coronal planes. Visualization of the diverticular neck was possible in five cases in the axial plane and in all 11 cases in coronal plane. Wiesner et al (6) examined retrospectively the incidence and volume of PAD in patients undergoing abdominal MDCT. Overall 1010 patients were covered. The average diameter of the diverticula was 17 $\mathrm{mm}$, ranging from 4 to $45 \mathrm{~mm}$. In $75 \%$ of cases with non-calculus obstruction of the bile ducts, the PAD diameter was higher than the mean value, which led to speculation that $\mathrm{PAD}$ volume might be a predisposing factor. The mean transverse diameter of the PAD in our patient group was $16.85 \mathrm{~mm}$. The average volume of PAD was $3.25 \mathrm{~cm}^{3}$, ranging from 2.5 to $4 \mathrm{~cm} 3$ in $95 \%$ of patients. Importantly there was no significant correlation between the age of the patients and the volume of the diverticula.

Frequency of PAD based on autopsy series and ERCP according to different sources was reported between 5 and $32 \%$ (3, 6-10). The relatively wide variation among investigators could be explained with differences in age and
GETSOV P., et al. number of the cohort. A retrospective study with 8642 patients showed a PAD frequency of $7.2 \%$ (7). Zoepf et al reported a PAD incidence of $12 \%$ in a study which included 2925 patients (10). They also found that $14 \%$ of patients had more than one diverticulum. Yildirgan et al were established 51 PAD between 381 patients studied(13.4\%) (6). There is few published data for PAD incidence reported by cross sectional imaging methods (MRI, MDCT). Retrospective study by Wiesner et al (5) which included 1010 patients who underwent abdominal MDCT reported PAD frequency of $3.2 \%$.

Our team has previously published a PAD frequency assessed by ERCP of $23.8 \%$ in a study among 3259 patients. In the current cohort we found slightly less incidence of PAD $-15.1 \%$ bearing that $91 \%$ had single, while $9 \%$ of patients had two PAD. Based on this results we can affirm close incidence of PAD as reported previously.

Duodenal diverticulosis was associated with an increased incidence of biliary pathology presumably due to the sphincter Oddi dysfunction and consequent ascending bacterial infection from the duodenum (2-4, 610). Possible association of PAD and ductal gallstones was previously discussed. Zoepf et al (10) also reported a difference in the incidence of ductal stones $-46.0 \%$ in patients with PAD and $33.1 \%$ in patients without PAD. The multivariate analysis revealed PAD as a significant risk factor for choledocholithiasis $(\mathrm{p}=0.016)$. Furthermore, there was a significantly higher incidence of recurrent choledocholithiasis in patients with PAD $6.6 \%$ when compared to those without PAD $(1.4 \%, \mathrm{p}<0.01)$. We can confirm that there was a correlation between the PAD and choledocholithiasis $(\mathrm{p}=0.009)$. More importantly we found relationship between chronic cholecystitis and PAD $(\mathrm{p}=0.002)$, which is rarely commented previously.

\section{CONCLUSION}

The results of our study showed that MRCP has good sensitivity, specificity, positive and negative predictive value for diagnosing PAD as compared to ERCP. The mean transverse diameter of the PAD was $16.85 \mathrm{~mm}$ and the average volume was $3.25 \mathrm{~cm}^{3}$. Our findings confirmed correlations between PAD, age and diseases of the biliary tract. The patients with PAD were found to be about 9 years older than those without PAD. There was also a 
significant association of PAD with choledocholithiasis $(\mathrm{p}=0.009)$ and cholecystitis $(\mathrm{p}=0.002)$. Based on this study we could conclude that MRCP improves the diagnostic assessment of PAD and their relationship with biliary tract diseases. MRCP should be considered in patients with suspected duodenal diverticula.

\section{REFERENCES}

1. Chomel, J.B.L.,. Histoire de L'Academie Royale, Paris. Paris:L'Institut de France, Academie des Sciences; 37, 1710

2. Chen, Q., Li, Z., Li, S., Ding X, Liu, Z., Wu, C., Gong, J., Zuo. Diagnosis and treatment of juxta-ampullary duodenal diverticulum. Clin Invest Med; 33 (5): E298-E303.,2010.

3. Getsov, P., Dineva, S., Vladimirov, B.. Endoscopic Retrograde Cholangiopancreatography in Patients with Periampullary Diverticula: Analysis of 3259 Cases. J Med Sci Public Health, 3, 3542,2014.

4. Mahajan, S.K., Kashyap, R., Chandel, U.K., Mokta J., Minhas, SS..Duodenal diverticulum: Review of literature. Indian Journal of Surgery, 66, 3, 140-155, 2006.

5. Wiesner, W., Beglinger, Ch., Oertli, D., Steinbrich, W. Juxtapapillary duodenal diverticula: mdet findings in 1010 patients and proposal for a new classification.JBRBTR , 92, 191-194,.2009.

6. Yildirgan, M.I., Basoglu, M., Yimaz, I., Atamanalp, S., Balik, A.A., Aydinli, B., Ozturk, G..Periampullary Diverticula Causing Pancreaticobiliary Disease. Digestive Diseases and Sciences, 49, 1943$1945, .2004$.

7. Zajac, A., $\quad$ Soleck, R., Kruszyna, T., Krzeszowiak, J..Duodenal diverticula and choledocholithiasis in own material, Przegl Lek., 62(12), 1398-400, 2005.

8. Zhang, W.Z., Xu, M.C., Shi, Z.H, Yang, Y.L. Relationship between juxta papillary duodenal diverticula and biliopancreatic diseases in patients over 60 years old, Di Yi Jun Yi Da Xue Xue Bao, 23(9),952-3, 2003.
GETSOV P., et al.

9. Zhong, Y., Wu, Y.H., Ye, Z.S., Guo, M.Y., Cai, Z.P, Zhang, B.M,et al.. The discussion about the relationship between intraduodenal peri-ampullary diverticulum and gallstones. Fujian Med, 26,23-25, 2004.

10.Zoepf, T., Zoepf, D.S., Arnold, J.C. et al..The relationship between juxta papillary duodenal diverticula and disorders of the biliopancreatic system: analysis of 350 patients, Gastrointest Endosc, 54, 56-61, 2001.

11.Rosenthal, T.. Können Duodenal divertikel eine klinische Bedeutung erlangen?, Med Klein, 4, 1421, 1908.

12.Case, J.T. Diverticula of the small intestine otherthan Meckel's diverticulum. JAMA 75, 1463-1470, 1920.

13.Ingilizova, I., Vassileva, J., Rupova, I., Pavlova, A. Radiation protection and safety in medical use of ionizing radiation in Republic of Bulgaria - harmonization of the national legislation with Euratom directives; Radiation Protection Dosimetry, 117,260-262, 2005.

14.Macari, M., Lazrus, D., Israel, G., Megibow, A..Duodenal diverticula mimicking cistic neoplasm of pancreas: CT and MR imaging findings in seven patients. AJR,180,195-199, 2003.

15.Morita, S., Ueno, E., Masukawa, A., Suzuzki, K., Machida, H., Fujimura, M..Defining juxtapapillary diverticulum with 3D segmented true FISP MRCP: comparison with conventional MRCP sequences with an oral negative contrast agent. Japanese Journal of Radiology. 27,423-429, 2009.

16.Perdikakis, E., Chryssou, E.G., Karantanas, A.. Diagnosis of periampullary duodenal diverticula: the value of new imaging techniques. Ann Gastroenterol; 24, 192199,2011.

17. Mazziotti, S., Costa, C., Ascenti, G., Gaeta, M., Pandolfo, A., Blandino, A. MR Cholangiopancreatography Diagnosis of Juxtapapillary Duodenal Diverticulum Simulating a Cystic Lesion of the Pancreas: Usefulness of an Oral Negative Contrast Agent. AJR, 185, 432-434,2005. 